\title{
QUANTUM COMPUTATION WITH HARMONIC OSCILLATORS
}

\author{
STEPHEN D. BARTLETT AND BARRY C. SANDERS \\ Department of Physics, Macquarie University, Sydney, New South Wales 2109, Australia \\ E-mail: Stephen.Bartlett@mq.edu.au; Barry.Sanders@mq.edu.au \\ BENJAMIN T. H. VARCOE \\ Max-Planck-Institut für Quantenoptik, 85748 Garching, Germany \\ E-mail: B.Varcoe@mpq.mpg.de \\ HUBERT DE GUISE \\ Faculté Saint-Jean, University of Alberta, 8406 rue Marie-Anne Gaboury, Edmonton, Alberta, \\ Canada T6C $4 G 9$ \\ E-mail: hdeguise@gpu.srv.ualberta.ca
}

\begin{abstract}
By encoding a qudit in a harmonic oscillator and investigating the $d \rightarrow \infty$ limit, we give an entirely new realization of continuous-variable quantum computation. The generalized Pauli group is generated by number and phase operators for harmonic oscillators. We describe a physical realization in terms of modes in a microwave cavity, coupled via a standard Kerr nonlinearity.
\end{abstract}

\section{Introduction}

The use of continuous-variable (CV) quantum computing allows information to be encoded and processed much more compactly and efficiently than with discrete-variable (qubit) computing. With CV realizations, one can perform quantum information processes using fewer coupled quantum systems: a considerable advantage for the experimental realization of quantum computing. The rapidly developing field of CV quantum information theory has applications to quantum error correction 1, quantum cryptography and quantum teleportation 3 , including an experimental realization of CV quantum teleportation 4 .

The most common proposed realization of CV quantum computation at present employs position eigenstates as a computational basis 1 ; these states are approximated experimentally using highly squeezed states 3 . Other CV realizations are described by a generalized Pauli group generated by the number operator $\hat{N}$ and a phase operator $\hat{\theta}$, and computational bases given by either harmonic oscillator number eigenstates or phase eigenstates 5 . These realizations are obtained formally by taking the $d \rightarrow \infty$ limit of the qudit, the $d-$ dimensional generalization of the qubit, and are important for five key reasons: $(i)$ these CV realizations are entirely distinct from the position eigenstate computational basis realization, both in terms of the computational basis and in terms of the SUM gate; (ii) the SUM gate employs a standard Kerr optical nonlinearity to couple two modes; (iii) these realizations give natural extensions of the qubit-based (discrete-variable) Pauli group, with a well-defined limiting procedure; $(i v)$ this CV quantum computation presents an appealing realization in terms of coupled modes in a microwave cavity using the powerful methods of state preparation 6 in such cavities: and $(v)$ these realizations give a new implementation of the well-studied phase operator 0 . 


\section{CV Quantum Computation using Harmonic Oscillators}

To describe CV quantum computation, one requires aninfinite-dimensional Hilbert space $\mathcal{H}$ and a representation of the generalized Pauli group 1 that serves as a group of unitary transformations on $\mathcal{H}$. By defining $\mathcal{H}$ to be the Hilbert space for a harmonic oscillator, one can define specific representations of the generalized Pauli group in terms of operators that act on the harmonic oscillator Hilbert space.

The natural generalization of the Pauli group 0 is the group generated by the number operator $\hat{N}$ and the (Pegg-Barnett $\mathrm{U}$ ) phase operator $\hat{\theta}$. Note that it is convenient to work with the finite-dimensional Hilbert space $\mathbb{H}_{d}$ of boson number not greater than $d-1$. On this Hilbert space, the phase operator is well-defined, and the system incorporates a finite energy cutoff and corresponding phase resolution.

With this generalization of the Pauli group, there are two natural computational bases. The first, the number state basis, consists of the harmonic oscillator energy eigenstates

$$
\hat{N}|n\rangle=n|n\rangle, \quad n=0,1, \ldots, d-1 .
$$

The second basis, the phase state basis, consists of eigenstates of the phase operator

$$
\hat{\theta}|\phi\rangle=\phi|\phi\rangle, \quad \phi=0, \frac{2 \pi}{d}, \ldots, \frac{2(d-1) \pi}{d} .
$$

These bases are "dual" in the sense that $\langle\phi \mid n\rangle=\exp (i \phi n)$.

To perform universal CV quantum computation $\mathbf{6}$, it is necessary to be able to: $(i)$ initially prepare a qudit in an arbitrary state for computation; (ii) realize an arbitrary unitary transformation on a single qudit; (iii) have a controlled two-qudit interaction gate such as the SUM gate 1

$$
\mathrm{SUM}:\left|s_{1}\right\rangle_{1} \otimes\left|s_{2}\right\rangle_{2} \mapsto\left|s_{1}\right\rangle_{1} \otimes\left|s_{1}+s_{2}\right\rangle_{2}
$$

and $(i v)$ perform von Neumann measurements in the computational basis.

\section{Proposed Realization as Coupled Modes of a Microwave Cavity}

One approach to physically realizing CV (qudit-based) quantum computation is by using a multimode microwave cavity. A single qudit is realized as the state of a (longitudinal) mode, and qudit-qudit interactions are performed by appropriately coupling different modes. By using a microwave cavity, one can take advantage of the recent technical advances of the micromaser: a micromaser provides a physical realization of the Jaynes-Cummings model, which describes the coupled system consisting of a single two-level atom and a single mode of the radiation field. Single atom interactions enable state preparation, qudit transformations, two-qudit coupling, and state measurement. Current high- $Q$ microwave cavities allow photon lifetimes up to $0.3 \mathrm{~s}$, several orders of magnitude larger than the atom interaction time.

State preparation in a microwave cavity is highly developed, both theoretically and experimentally. Proposals $\mathrm{a}$ for preparation of an arbitrary state of the radiation field involve injecting a sequence of atoms and are conditional on measurement of the output atoms; a deterministic approach to the preparation of Fock states (not involving conditional measurements) has been experimentally realised 6 . Recently, it has been proposed 10 that any state of the radiation field can be prepared with arbitrarily high fidelity using a sequence

QCProc150101: submitted to Rinton on October 29, 2018 
of entangled atoms; this technique has the important features that the resulting state of the field is not entangled with that of the atoms, and that the method is independent of the initial state of the field.

An arbitrary unitary transformation on a single qudit (single mode), to any desired precision, can be performed efficiently using a combination of phase-space displacements, squeezing, and a nonlinear Kerr effect 6 . By an appropriate combination of these transformations, one can approximate (to arbitrary accuracy) any polynomial Hamiltonian in $\hat{a}^{\dagger}$ and $\hat{a}$.

Linear transformations of a cavity mode state may be performed by coupling the mode to an external monochromatic coherent field via transmission through one of the mirrors 11. A strong coherent field is directed into the cavity through a low-transmissivity mirror, and the result on the mode state is that it is displaced; i.e., the field state is acted upon by a unitary Glauber displacement operator. The choice of frequency for the incoming coherent field determines which of the longitudinal field states (each with a different frequency) is displaced.

Squeezing the field state requires a nonlinear process, as does the nonlinear Kerr transformation of the field state. The squeezing transformation may be achieved via a $\chi^{(2)}$ nonlinearity and the latter by a $\chi^{(3)}$ nonlinearity. A nonlinearity is effected by passing atoms through the cavity one at a time: during the time of passage, the field states undergoes a nonlinear evolution. An effective nonlinearity is obtained by adiabatically eliminating the slowly varying atomic degrees of freedom. The field in the cavity may be squeezed by injecting two-level atoms into a micromaser cavity 12 or three-level atoms 13 . By choosing atomic levels and motional parameters carefully, the squeezing (Bogoliubov) transformation can be approximated. The Kerr, or $\chi^{(3)}$, nonlinearity is generally smaller, but it is possible to employ a four-level atom, passing through the cavity, to obtain a large Kerr nonlinearity 14. Thus, by directing atoms through the cavity, unitary $\chi^{(2)}$ and $\chi^{(3)}$ evolutions particular field states can be effected. The Kerr nonlinearity can also effect a cross-phase modulation to perform the micromaser equivalent of the optical cavity quantum electrodynamics conditional phase shift $\mathrm{t}$.

For quantum computation, we must also realize a gate that performs a two-qudit interaction. Consider two oscillators coupled by the four-wave mixing interaction Hamiltonian $\chi \hat{N}_{1} \hat{N}_{2}=\chi \hat{a}_{1}^{\dagger} \hat{a}_{1} \hat{a}_{2}^{\dagger} \hat{a}_{2}$. This Hamiltonian for an optical system describes a four-wave mixing process in which $\chi$ is proportional to the third-order nonlinear susceptibility 16 . Let oscillator 1 be in a state $\left|s_{1}\right\rangle_{1}$ encoded in the number state basis, and let oscillator 2 be in a state $\left|s_{2}\right\rangle_{2}$ encoded in the phase state basis. This interaction Hamiltonian generates the transformation

$$
e^{-\mathrm{i} \chi \hat{N}_{1} \hat{N}_{2} t}\left|s_{1}\right\rangle_{1} \otimes\left|s_{2}\right\rangle_{2}=\left|s_{1}\right\rangle_{1} \otimes\left|\chi t s_{1}+s_{2}\right\rangle_{2} .
$$

Thus, with time $t=\chi^{-1}$, this Hamiltonian generates the SUM transformation.

Quantum computation with multiple qudits could be performed by coupling several modes in a single cavity; each mode realizes a single qudit. Modes are coupled via a SUM interaction of the time described above. Note that the control qudit for the sum operation must be encoded in the number state basis, and the target qudit must be in the phase state basis

Measurements in the number state computational basis can be performed using a QND measurement of photon number 17 . Atoms passing through the cavity have their momen- 
tum coupled to the radiation field; the momentum distribution of outgoing atoms yields a measurement of photon number.

In summary, we have presented a new form of continuous variable computation in terms of number and phase operators, and describe a realization in terms of coupled modes of a microwave cavity using linear transformations, squeezing, and nonlinear Kerr media. This new approach has the advantage over position-eigenstate CV computation in that the computational basis states, for large but finite $d$, are well-defined and obtainable, and do not require "infinite-squeezing" of Gaussian wavepackets.

\section{Acknowledgments}

This project has been supported by an Australian Research Council Large Grant and by

a Macquarie University Research Grant. We acknowledge helpful discussions with Samuel Braunstein and Terry Rudolph.

\section{References}

1. D. Gottesman, A. Kitaev, and J. Preskill, Phys. Rev. A 64, 012310 (2001).

2. T. C. Ralph, Phys. Rev. A 61, 010303(R) (2000).

3. S. L. Braunstein and H. J. Kimble, Phys. Rev. Lett. 80, 869 (1998).

4. A. Furusawa, J. L. Sørensen, S. L. Braunstein, C. A. Fuchs, H. J. Kimble, and E. S. Polzik, Science 282, 706 (1998).

5. S. D. Bartlett, H. de Guise and B. C. Sanders, Phys. Rev. A (in press) (2002) quant-ph/0109066.

6. K. Vogel, V. M. Akulin, and W. P. Schleich, Phys. Rev. Lett. 71, 1816 (1993).

7. D. T. Pegg and S. M. Barnett, J. Mod. Optics 44, 225 (1997).

8. S. Lloyd and S. L. Braunstein, Phys. Rev. Lett. 82, 1784 (1999).

9. S. Brattke, B. T. H. Varcoe and H. Walther, Phys. Rev. Lett. 86, 3534 (2001); B. T. H. Varcoe, S. Brattke, M. Weidinger, and H. Walther, Nature 403, 743 (2000); B. T. H. Varcoe, S. Brattke and H. Walther, J. Opt. B: Quantum Semiclass. Opt. 2, 154 (2000).

10. T. Wellens, A. Buchleitner, B. Kümmerer, and H. Maassen, Phys. Rev. Lett. 85, 3361 (2000).

11. C. T. Bodendorf, G. Antesberger, M. S. Kim, and H. Walther, Phys. Rev. A 57, 1371 (1998).

12. F. L. Kien, Phys. Rev. A 44, 3282 (1991).

13. M. Orszag, R. Ramírez, J. C. Retamal and L. Roa, Phys. Rev. A 45, 6717 (1992); R. R. Puri, C. K. Law and J. H. Eberly, Phys. Rev. A 50, 4212 (1994)

14. S. Rebić, S. M. Tan, A. S. Parkins, and D. F. Walls, J. Opt. B: Quantum Semiclass. Opt. 1, 490 (1999).

15. Q. A. Turchette, C. J. Hood, W. Lange, H. Mabuchi, and H. J. Kimble, Phys. Rev. Lett. 75, 4710 (1995).

16. G. J. Milburn and D. F. Walls, Phys. Rev. A 28, 2065 (1983).

17. M. J. Holland, D. F. Walls, and P. Zoller, Phys. Rev. Lett. 67, 1716 (1991).

QCProc150101: submitted to Rinton on October 29, 2018 